\title{
Identificación de problemas de salud como efecto del sedentarismo: Un estudio con personas dedicadas a la docencia durante la pandemia covid19
}

\author{
Identification of health problems as an effect of sedentary lifestyles: A \\ study of teachers during the covid19 pandemic.
}

\author{
Medina Paredes Segundo Víctor. ${ }^{1}$, Flores Robalino Rosita Gabriela. ${ }^{2}$, Villalba Garzón \\ Gabriela Alexandra. ${ }^{3} \&$ Barrera Cueva Janeth del Carmen. ${ }^{4}$
}

\begin{abstract}
.
Introduction. Of the various professions, teaching is one of the most exciting, the fact of sharing experiences and knowledge, learning from others is part of the essence of the human being, making it a way of life requires vocation and full-time dedication, but there are certain health problems associated with sedentary lifestyles. Objective. The study was to identify the health problems as an effect of sedentary lifestyle in people dedicated to secondary teaching; however, due to the problem of the pandemic covid19 the teacher spends several hours a day in telework activities, an average of 8 to 12 hours, of which teachers are dedicated to fulfill their pedagogical work and, in almost all activities remain seated, physically inactive. Methodology. The study was developed through a

\footnotetext{
${ }^{1}$ Universidad Técnica de Ambato, Facultad de Ciencias Humanas y de la Educación, Carrera de Pedagogía de la Actividad Física y Deporte. Ambato, Ecuador, email sv.medina@uta.edu.ec

${ }^{2}$ Universidad Técnica de Ambato, Facultad de Ciencias Humanas y de la Educación, Carrera de Pedagogía de la Actividad Física y Deporte. Ambato, Ecuador, email rg.flores@uta.edu.ec ORCID. https://orcid.org/0000-0002-4083-585

${ }^{3}$ Universidad Técnica de Ambato, Facultad de Ciencias Humanas y de la Educación, Carrera de Pedagogía de la Actividad Física y Deporte. Ambato, Ecuador, email ga.villalba@uta.edu.ec ORCID. https://orcid.org/0000-0001-5589-4927

${ }^{4}$ Universidad Técnica de Ambato, Facultad de Ciencias Humanas y de la Educación, Carrera de Pedagogía de la Actividad Física y Deporte. Ambato, Ecuador, email jdc.barrera@uta.edu.ec ORCID. https://orcid.org/0000-0002-1985-9478
} 
quantitative methodology with a descriptive approach; the research worked with 104 education professionals of the Atahualpa Educational Unit of the city of Ambato, two groups were established according to gender, 66 women and 38 men; the instrument for the collection of information was based on a structured questionnaire applied through technological tools. Results. The main results show that teachers present various health problems such as hypertension, diabetes, intestinal and gastric disorders, kidney problems, bone system and stress. Conclusions. The data obtained allow, as far as possible, the proposal of viable alternatives for the prevention of integral health and the practice of physical activity in teachers.

Keywords: health problems, sedentary lifestyle, teaching, prevention.

\section{Resumen.}

Introducción. De las diversas profesiones, la docencia es una de las más apasionantes, el hecho de compartir experiencias y conocimientos, aprender de otros es parte de la esencia del ser humano, hacer de ello una forma de vida requiere de vocación y dedicación a tiempo completo, pero existe ciertos problemas de salud asociados al sedentarismo. Objetivo. El estudio fue identificar los problemas de salud como efecto del sedentarismo en las personas dedicadas a la docencia secundaria; sin embargo, por el problema de la pandemia covid19 el docente pasa varias horas al día en actividades de teletrabajo, un promedio de 8 a 12 horas, de las cuales los docentes se dedican a cumplir con su labor pedagógica y, en casi todas las actividades permanecen sentados e inactivos físicamente. Metodología. El estudio se desarrolló mediante una metodología cuantitativa con un enfoque descriptivo; en la investigación se trabajó con 104 profesionales de Educación de la Unidad Educativa Atahualpa de la ciudad de Ambato, se establecieron dos grupos, en función del género, 66 personas mujeres y 38 varones; el instrumento para la recolección de la información se basó en un cuestionario estructurado aplicado mediante herramientas tecnológicas. Resultados. Los principales resultados muestran que los docentes presentan diversos problemas de salud como la hipertensión, diabetes, afecciones intestinales, gástricos, problemas renales, sistema óseo y estrés. Conclusiones. Los datos obtenidos permiten en lo posible el planteamiento de alternativas viables para la prevención de la salud integral y práctica de actividad física en los docentes.

Palabras claves: problemas en la salud, sedentarismo, docencia, prevención.

\section{Introducción.}

La labor docente estudia varios aspectos relevantes para identificar, evaluar y perfeccionar su desempeño, mediante programas de formación docente. A través de información bibliográfica concerniente a prácticas docentes, identificamos algunas recomendaciones para ser un buen profesor, realizamos indagaciones referentes al tema de estudio; salud preventiva y los desaciertos de la práctica docente en la salud. Sin 
embargo, en un entorno de investigación, debe pasar de las practicas prescritas a las practicas reales. Construir las prácticas docentes como objetivos de aprendizaje significa comprender tanto las referencias conceptuales como las diversas formas de utilizarlas: los marcos metodológicos, enfoques, métodos, diseños e instrumentos utilizados, sin embargo, la práctica docente va repercutiendo en la salud, concordamos con (GuevaraManrique, A. C., Sánchez-Lozano, C. M., \& Parra, L. 2014) quienes mencionan que el estrés laboral es una enfermedad peligrosa que afecta a los docentes en su salud física y mental.

La docencia se define como un conjunto de guías basadas en una finalidad formativa específica y mediante la conexión subjetiva con los estudiantes, que constituye un conjunto de conductas que establecen el trabajo diario de un docente. Estas prácticas están influenciadas por el conocimiento, las ideas y las creencias de los profesores (Barrón, 2015) durante la labor docente; por otro lado, están los ámbitos de autoeficacia docente para optimizar la gestión en el aula con miras a implicar al estudiante en el proceso de aprendizaje (Rodríguez, 2009). La docencia es una actividad profesional muy necesaria para promover el progreso social, sin embargo, en esta práctica existen algunos factores que inciden en la salud, uno de ellos es un típico estilo de vida sedentario para las actividades docentes. La mayoría de las personas que se dedican a esta actividad necesitan pasar mucho tiempo (entre 6 y 8) sentadas todos los días, realizando actividades de investigación, planificación y evaluación (Cazau, 2011).

Desde la década de 1980, las investigaciones han demostrado que existe una conexión biológica entre la docencia y diversas enfermedades de la salud (cardiovascular, respiratoria, lumbalgia, dolor cervical, preeclampsia o úlcera gástrica, etc.), así como psicológica (ansiedad, depresión, insatisfacción laboral, disminución de la productividad, absentismo, emociones negativas fuera del trabajo).

En el campo de la enseñanza, hay mucha discusión sobre el estrés y los efectos del "agotamiento" (también llamado "síndrome burnout", "síndrome de estrés laboral de enfermería", "síndrome de agotamiento profesional"), aunque los términos están estrechamente relacionados su significado es diverso, no es lo mismo estar estresado que con agotamiento. El concepto de "agotamiento" fue propuesto por Freudenberger en 1974. Posteriormente, Maslach y Pines (1977) dieron a conocer ampliamente su terminología. Desde entonces, el término se ha utilizado para referirse al agotamiento laboral que sufre el personal de servicios (educación, salud, administración pública), etc.), debido a que las condiciones laborales tienen fuertes efectos sociales (Cazau, 2011). El estrés laboral debe ser estudiado desde la percepción y vivencias de la práctica docente, visto en su conjunto integrador y analizado desde 4 aspectos fundamentales: el estrés laboral, el docente, el estudiante y el ámbito institucional (Rodríguez Ramírez, 2017). Cuando una persona está sujeta a requisitos de comportamiento estrictos, tendrá un impacto en la salud. La respuesta de estímulo al estrés se produce de inmediato, una vez superada esta situación, el cuerpo se activará y reequilibrará, pero si se repite con demasiada frecuencia, el cuerpo se desgastará, el efecto burnout se produce cuando sobrepasan su capacidad de reacción de una forma adaptativa. Aparece con síntomas de 
fatiga, cansancio y agotamiento mental, acompañados de una pérdida de energía severa, lo que lleva a una disminución en el número y calidad de las actuaciones positivas. En general, es una sensación asociada a la depresión, fracaso y actitudes negativas hacia el trabajo, la vida y las actividades sociales.

Los ejercicios físicos regulares para adaptarse a las capacidades y características personales de las personas tienen un efecto beneficioso sobre la salud orgánica y física Castro, (2014), además existe documentación científica que la actividad física reporta beneficios en la dimensión física y también contribuye a mejorar la dimensión psíquica y social en la salud de las personas (Cuadarado, 2017). La actividad física se define como cualquier movimiento físico producido por el músculo esquelético que requiere un gasto energético. Por tanto, la actividad física no debe confundirse con el deporte. Se trata de una variedad de actividades físicas planificadas, organizadas y repetitivas cuyos objetivos están relacionados con la mejora o el mantenimiento de uno o más componentes de la salud física. (Cáceres, 2014). La práctica física abarca el ejercicio, pero también otras actividades como movimiento corporal y se realizan como parte de los momentos de juego, del trabajo, de formas de transporte activas, de las tareas domésticas y de actividades recreativas; en la actividad laboral (docencia) también debe estar presente la actividad física. También debe tenerse en cuenta que los beneficios psicológicos del ejercicio físico están estrechamente relacionados con la capacidad de resistir el estrés de manera más eficaz y desarrollar hábitos de salud protectores. (Bonilla, 2013). Mejora la autoestima y el auto concepto, disminuye el riesgo de adquirir otros tipos de enfermedades y estrés, actuando como efectos antidepresivos y tranquilizantes, mejora en la coordinación y reflejos, previene el insomnio, regula los ciclos de sueño y produce mejores resultados en procesos de socialización. Por consiguiente, Sánchez-González, (2018) afirma que la realización de ejercicio físico ayuda a la prevención del deterioro cognitivo en las personas.

La actividad física dependerá de la etapa de la vida en la que está la persona: en sus inicios es el juego, en la juventud es el deporte y en la adultez se combinan estas experiencias en diferentes formas de expresión y desarrollo físico y mental. Los docentes son uno de los grupos más vulnerables que tienen mayor riesgo de padecer sedentarismo, por el tiempo que pasan sentados desarrollando las diferentes actividades, como son las académicas, comunitarias, administrativas, y hoy en día las clases en línea. Las diferentes posturas inadecuadas del sedentarismo, son las causantes de muchos problemas de salud especialmente de columna, además existen otros factores como la monotonía, la rutina, el manejo inadecuado del tiempo, el agotamiento pueden ayudar que empeore los cuadros de este tipo de molestias y termine afectando la salud de los docentes, las grandes cantidades de horas, esfuerzos físicos y psicológicos pueden aparecer desencadenando estrés laboral, que afecta notoriamente la calidad de vida y el bienestar personal (Montoya, 2017).

Las instituciones educativas deben jugar un papel activo y superior y así formar alianzas estratégicas para cambiar los comportamientos de sedentarismo en los docentes y el sector educativo; para ello se debe aplicar normativas o planes de hábitos de salud, uno de ellos 
puede ser el ejercicio físico regular durante la jornada laboral, este tipo de actividad contribuirá a mejorar la salud y el trabajo de los docentes, especialmente en las relaciones socio educativas (Hidalgo,2017). Teniendo esto en cuenta, es necesario determinar el tiempo que dedica el docente al ejercicio físico y se mantiene de pie, sentado, caminando o realizando trabajos pesados, con este diagnóstico en las instituciones se puede establecer un lugar de trabajo adaptado para poder tener intervalos de descanso durante la jornada laboral o mantener un programa de ejercicio físico específico en el lugar de trabajo para todos los docentes. Un estilo de vida sedentario se define como la no realización de ejercicio físico mínimo 30 minutos a la semana, pasar sentado o acostado el mayor tiempo posible, tener periodos de tiempo en los que se lleva a cabo actividades que consumen muy poca energía. Al definir este marcador de riesgo, es importante destacar que el sedentarismo es uno de los cuatro factores de mayor riesgo de muerte debido al comportamiento sedentario en las actividades laborales y domésticas (Mocha-Bonilla Julio, A., Alava, D. J. H., Muñoz, J. M. V., \& Castro, N. J. V. 2018). En definitiva, un estilo de vida sedentario es un problema de salud pública que amerita ser estudiado en todos los ámbitos.

La actividad física para los adultos entre 18 y 64 años, presenta tres criterios para considerar a las personas activas, se debe realizar al menos 150 minutos de actividad física moderada por semana, o 75 minutos de actividad física vigorosa por semana, o puede ser una combinación de ejercicio de intensidad moderada y vigorosa que supere el consumo mínimo de energía (Barrera, R. 2017). La Organización Mundial de la Salud (OMS) define a la actividad física como cualquier movimiento corporal producido por los músculos esqueléticos, con el debido consumo de energía. Todo ello incluye las actividades de la vida diaria, las actividades al jugar, trabajar, viajar y actividades recreativas. Ante esta realidad, el estudio tiene como objetivo identificar algunos de los problemas de salud provocados por el sedentarismo en un grupo de docentes que imparten clases virtuales durante la pandemia covid19.

\section{Metodologia.}

La metodología empleada fue de tipo cuantitativa debido a que describen las variables de estudio, por el uso de datos numéricos que permitieron complementar la investigación; se realizó un análisis descriptivo observacional porque presenta las características del personal docente en cuanto a la frecuencia de actividad física y los problemas de salud que presentan producto del sedentarismo; es observacional ya que no se necesita a futuro realizar un seguimiento continuo o algún tipo de control permanente.

Participantes. El estudio se desarrolló con 104 docentes de la Unidad Educativa Atahualpa, los cuales están divididos en los diferentes niveles educativos, Educación Inicial, Educación General Básica y el Bachillerato. El personal docente se encuentra dividido en 38 hombres y 66 mujeres, es decir, se consideró a todo el personal docente de la institución; con ello se pudo obtener datos más confiables al investigar a toda la población docente. En primera instancia se analizó el tipo de actividades físicas que 
realizan, si efectúan alguna actividad física o practican algún tipo de ejercicio, para luego conocer que problemas de salud enfrentan los docentes durante la pandemia, para finalizar determinando si los problemas de salud de los últimos seis meses son producto de un comportamiento sedentario.

Instrumento. Para cumplir los protocolos establecidos por los organismos pertinentes fue necesario recopilar la información mediante la creación de un formulario electrónico (google forms), el cual fue enviado vía WhatsApp a todo el personal docente de la institución, por medio del formulario se obtuvo los datos y se recabó la información correspondiente para mitigar los efectos del contagio por motivos de la pandemia sanitaria covid 19; para ello se estructuró un cuestionario compuesto por 8 preguntas estructuradas.

Protocolo. Dada la situación de emergencia sanitaria por la que atraviesa el mundo, se mantuvo contacto telefónico con la Autoridad Institucional, la cual fue el primer contacto para empezar el proceso de investigación, posteriormente se pudo enviar mediante correo electrónico el oficio solicitando autorización para recopilar la información al personal docente. Con la acertada respuesta se procedió a enviar el enlace del formulario al docente responsable de Tecnologías de la Comunicación e Información de la institución, siendo la persona encargada de distribuir el formulario a todo el personal docente, una vez enviado el formulario, las respuestas se fueron registrando para su posterior análisis e interpretación, el objetivo fue analizar la información obtenida de los docentes objeto de estudio para fortalecer la prevención de problemas de salud mediante la práctica de actividad física.

\section{Resultados.}

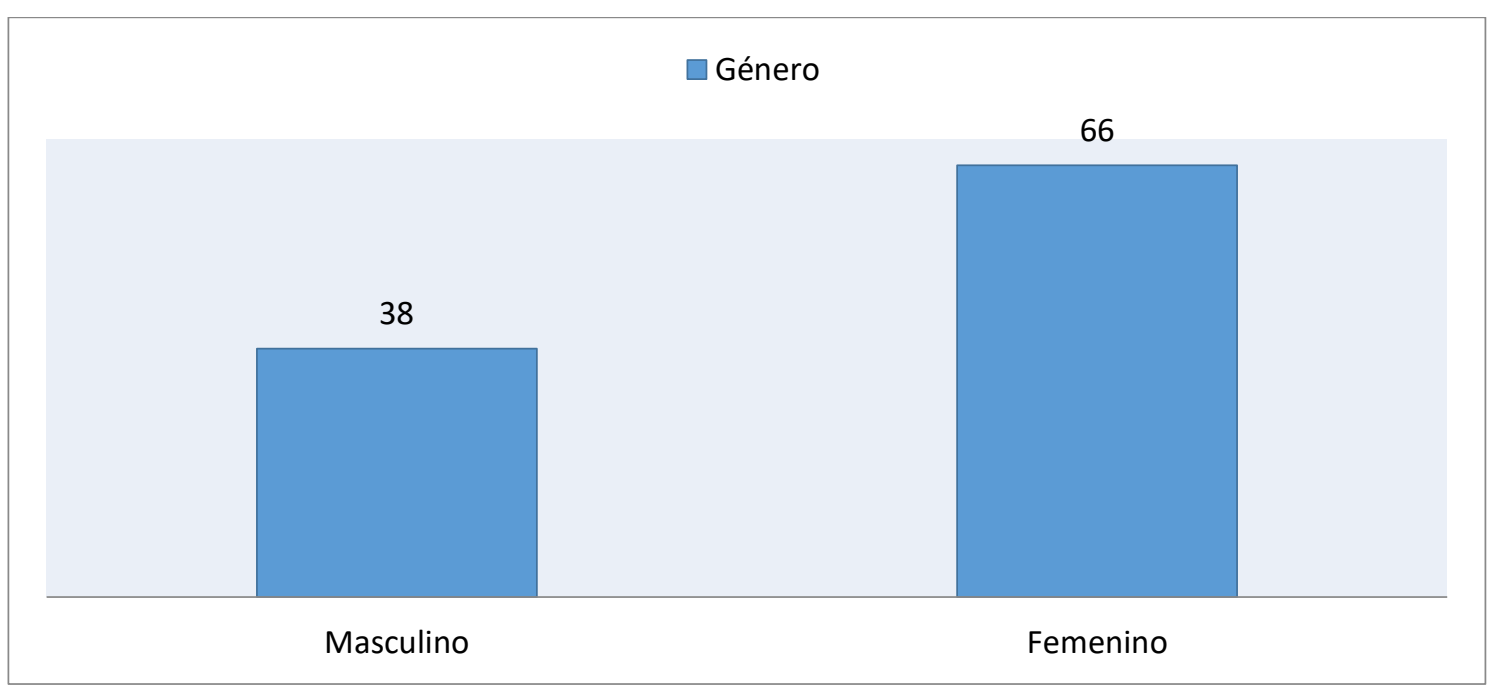

Figura 1 Género en los Docentes

Fuente: Elaborado por los investigadores

En la figura 1, se pueden observar que de los resultados obtenidos en el personal docente de la Unidad Educativa objeto de estudio, los datos presentados dan a conocer que existen 
38 docentes de género masculino y 66 docentes mujeres; por lo tanto, existe mayor índice de docentes de género femenino dedicadas a la docencia.

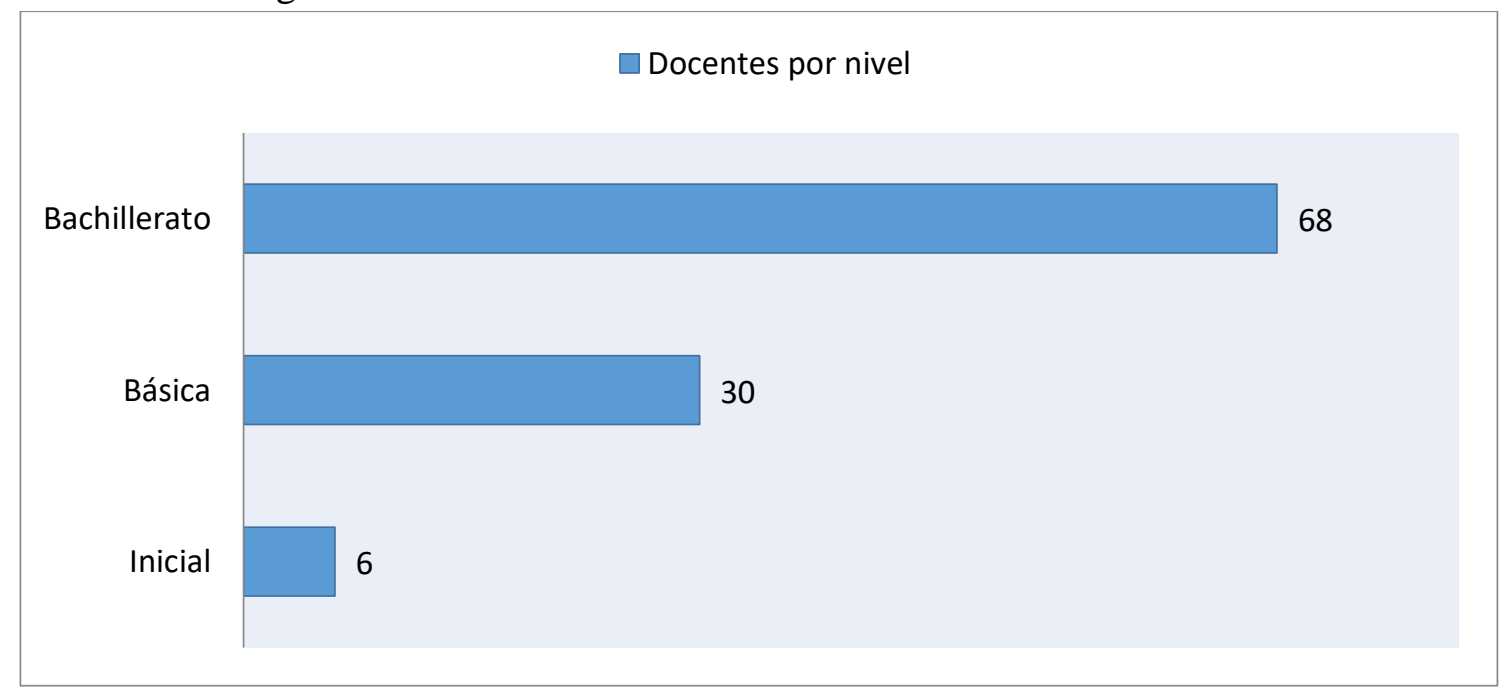

Figura 2 Participación Docentes por Nivel

Fuente: Elaborado por los investigadores

En la figura 2 se puede apreciar que la oferta académica educativa de la institución está dividida en tres niveles Inicial, Básica y Bachillerato; observándose 6 docentes para el Nivel Inicial, 30 maestros para Educación Básica y 68 para el bachillerato. Con lo cual se aprecia que la mayor cantidad de docentes se encuentran laborando en el Bachillerato.

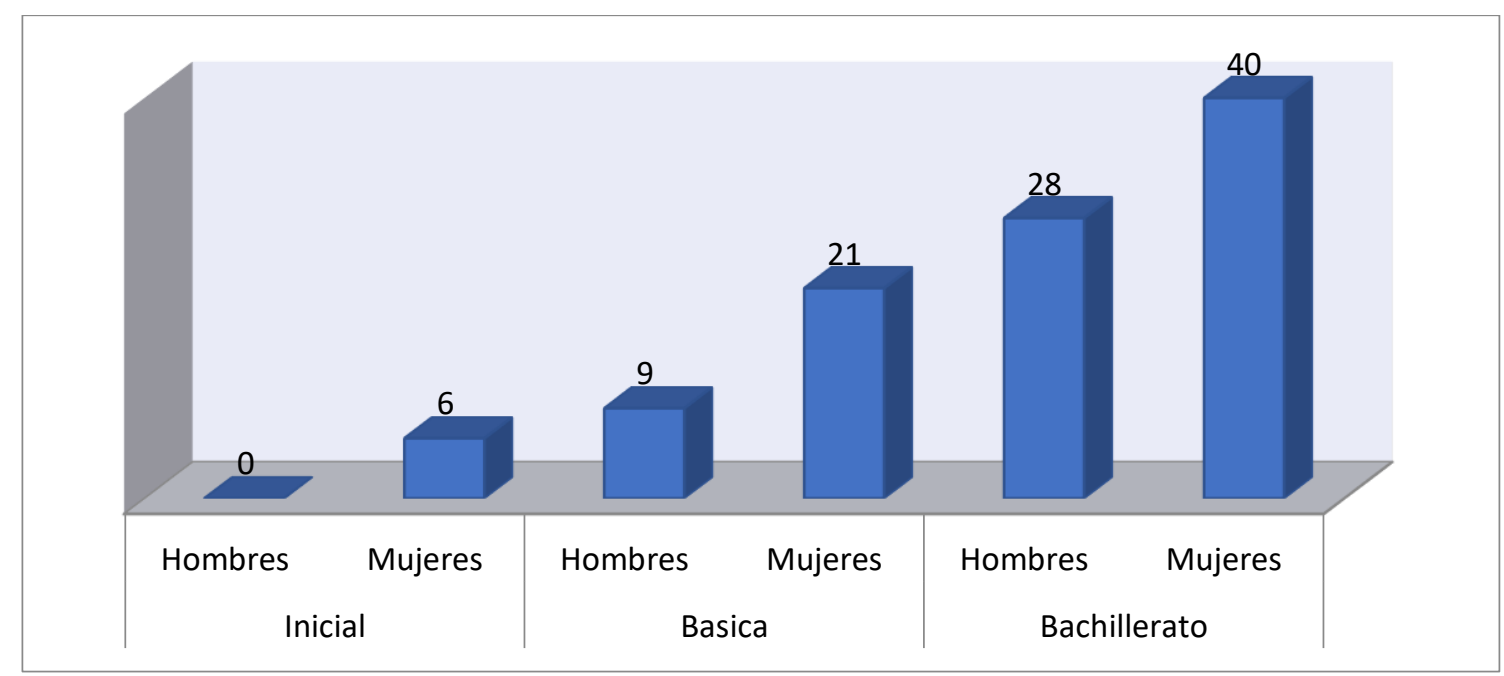

Figura 3 Docentes por niveles

Fuente: Elaborado por los investigadores

De acuerdo con la figura 3 se puede identificar a los docentes que realizan sus actividades de acuerdo al siguiente detalle: en Inicial existen 6 mujeres; en Educación Básica 9 hombres y 21 mujeres; en Bachillerato 28 varones y 40 mujeres. 


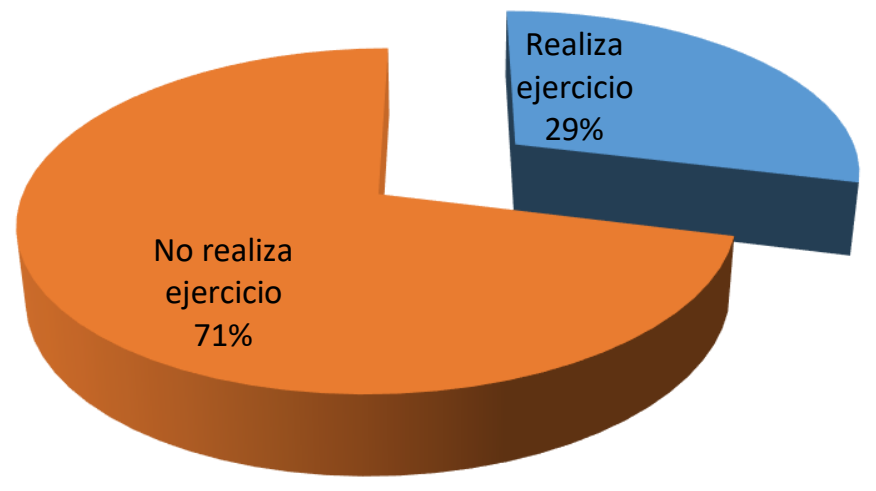

Figura 4 Realización de ejercicio en el personal docente Fuente: Elaborado por los investigadores

Los datos relacionados con la pregunta realizar algún tipo de ejercicio muestran que el $71 \%$ del personal docente no realiza ningún tipo de ejercicios ; mientras que el 29\% restante si realiza actividad física en su tiempo libre los datos presentados en la figura 4 muestran claramente que la mayoría del personal docentes presenta un comportamiento sedentario ya que no realizan actividad física, con lo cual es necesario que se implemente durante la jornada laboral pausas activas para mantener un hábito de prevención y cuidado de la salud integral en los docentes.

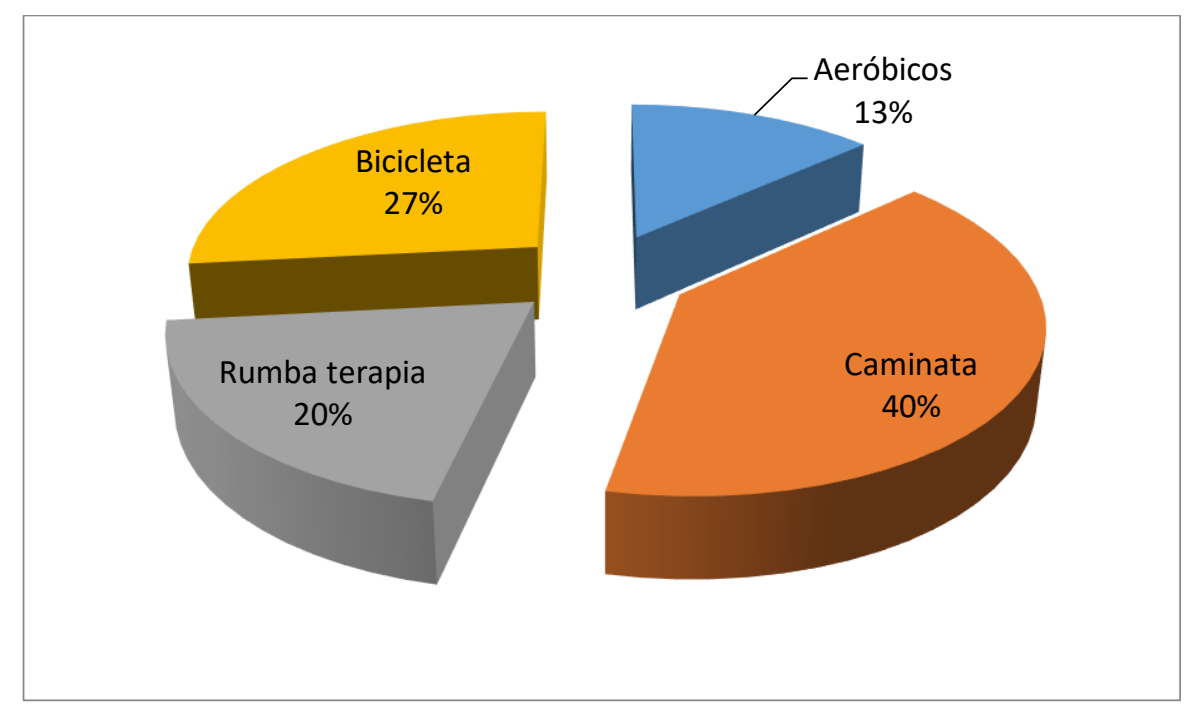

Figura 5 Tipo de ejercicio que realizan los docentes Fuente: Elaborado por los investigadores

Del 29\% de docentes encuestados, quienes contestaron que si realizan actividad física se puede desglosar que el $40 \%$ realizan caminatas moderadas, el $27 \%$ realiza actividades de ciclismo, el $20 \%$ se dedica a realizar rumba terapia y el finalmente el $13 \%$ realizan actividades físicas de tipo aeróbicas. Como se observa en la figura 5. 


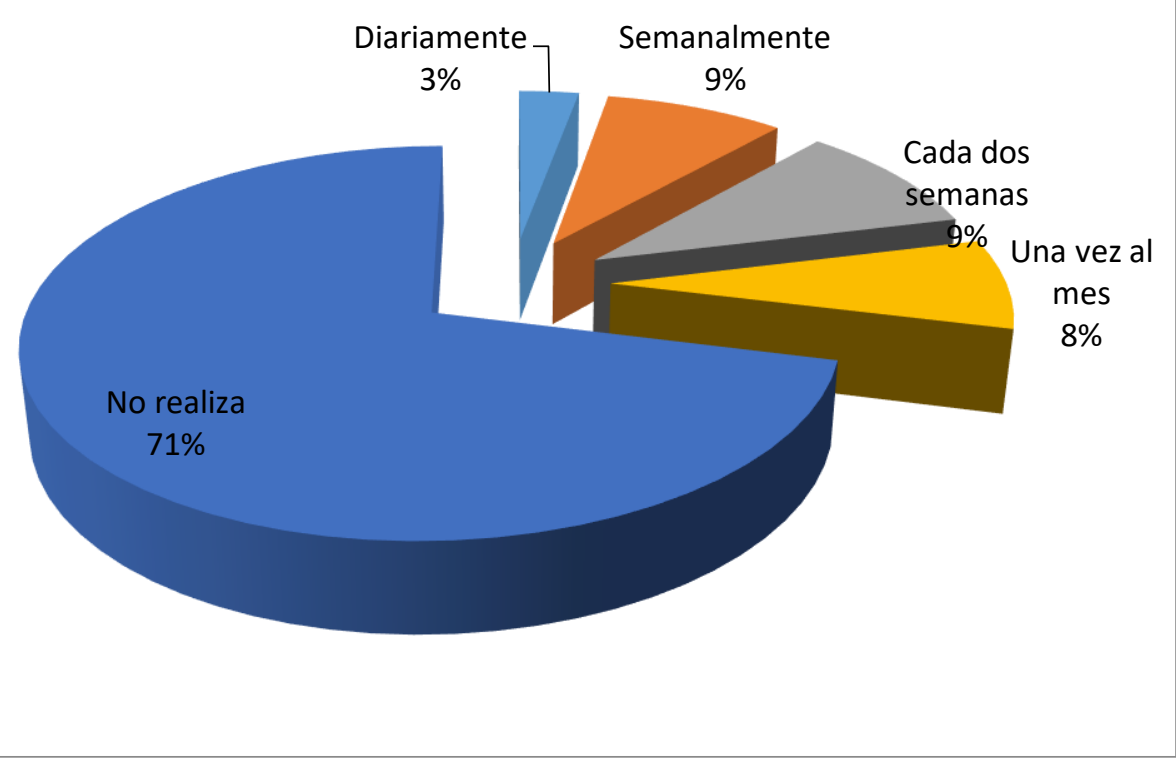

Figura 6 Frecuencia en la realización de ejercicio Fuente: Elaborado por los investigadores

La figura 6 demuestra que del 29\% de docentes encuestados el 9\% realizan actividades físicas semanales, el otro $9 \%$ realizan cada dos semanas el $8 \%$ una vez a la semana y tan solo el 3\% realizan actividad física diaria; mientras que el $71 \%$ de docentes de la institución no realizan actividad física, por consiguiente, el personal docente se encuentra dedicado a sus actividades academias curriculares, dejando de lado un programa de ejercicios para mantener su calidad de vida.

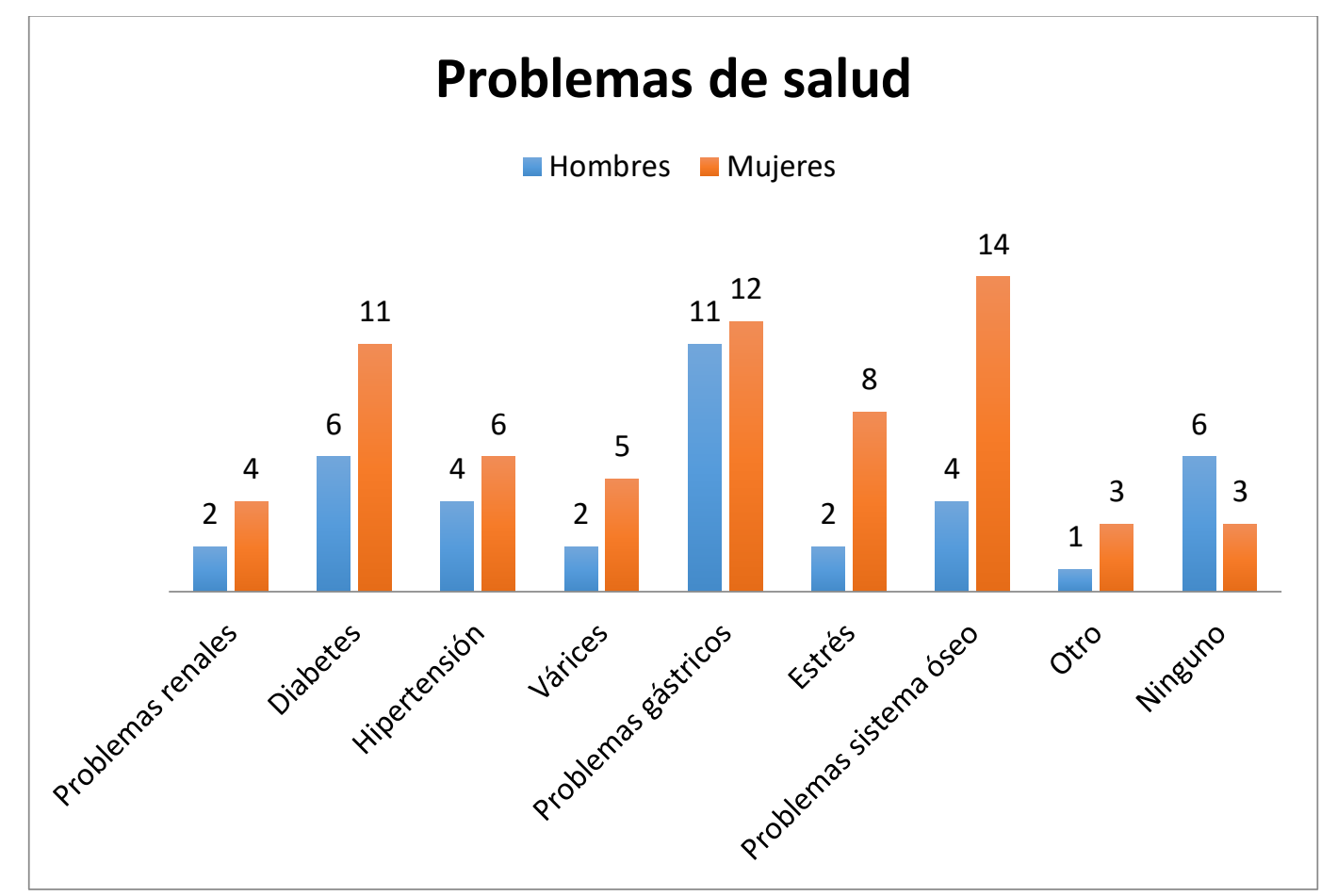

Figura 7 Problemas de salud presentes en el personal docente Fuente: Elaborado por los investigadores 
La figura 7 demuestra en los docentes encuestados los problema de salud que están presentando: 18 docentes presentan problemas en su sistema óseo, 23 docentes presentan problemas gástricos, 17 docentes se encuentran con problemas de diabetes, 10 docentes presentan problemas de hipertensión 10 docentes presentan problemas de estrés, 7 docentes presentan problemas de venas varices, 6 docentes con problemas renales, 4 docentes con otras afecciones y tan solo 9 docentes no tienen ningún problema de salud.

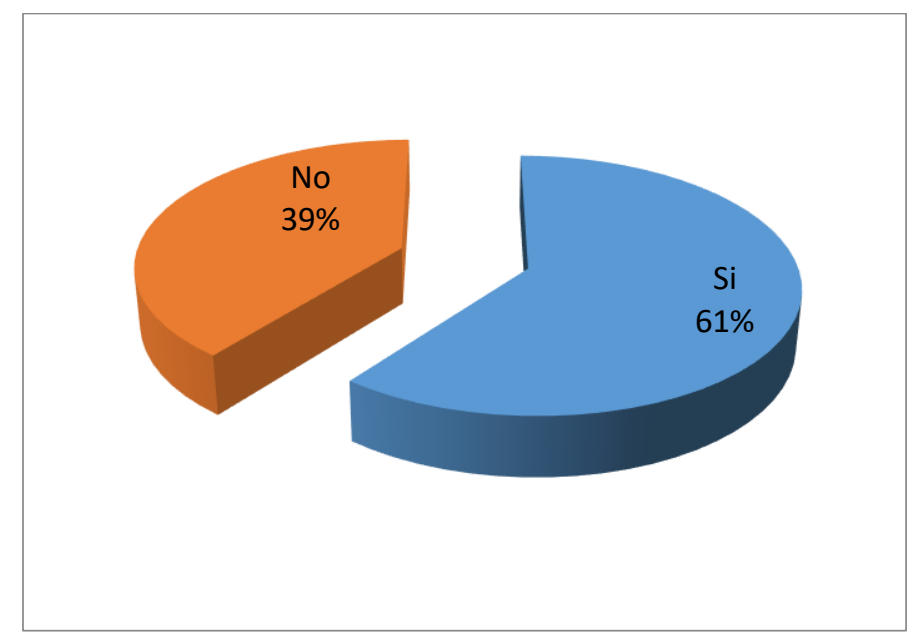

Figura 8 Estaría dispuesta/o a participar en algún programa de ejercicios para evitar el sedentarismo y mejorar su estado de salud.

Fuente: Elaborado por los investigadores

Finalmente, en la figura 8 se puede observar que solo el $61 \%$ de encuestados del personal docente están de acuerdo en participar de un programa de ejercicios para mejorar su estado de salud, mientras que el 39\% mantienen una respuesta negativa, es decir, que no desean cambiar sus hábitos de vida sedentaria. Considerando que solo el $21 \%$ de los docentes se ejercitan de manera ocasional se debería fomentar una campaña para dejar de lado el estilo de vida sedentario e involucrar a todo el personal docente de la Institución a mejorar su salud.

\section{Conclusiones.}

- En el Ecuador según el Ministerio de Salud (2012) las principales causas de muerte durante la década 2000 - 2010, han sido las enfermedades crónicas, que ocupan los primeros lugares de mortalidad, lo cual puede estar relacionado con sedentarismo y malos hábitos de nutrición. La encuesta ENSANUT-ECU 2011 2013 reporta que la prevalencia de actividad física global por sexo en adultos de 18 a < 60 años, es más frecuente en hombres (64.9\%) que en mujeres (46.2\%); y entre los determinantes de los problemas nutricionales en este grupo poblacional son dieta no saludable, estrés, alcohol, tabaco, drogas y sedentarismo. Por consiguiente, los dos informes ya mencionan al comportamiento sedentario como uno de los factores que afectan a la salud personal. De acuerdo con nuestros resultados la realidad analizada en el presente estudio (personal Docente de la 
Unidad Educativa Atahualpa) presenta algunos problemas en su salud asociados al comportamiento sedentario.

- Los datos analizados en el estudio presentan un interés marcado por el comportamiento sedentario, el cual está repercutiendo la salud de los docentes, es así que se propone un plan de actividad física y pausas activas para mitigar los inconvenientes de salud, involucrar a todos quienes se dedican a la actividad docente.

- Para las actividades diarias, todas las personas deben considerar la importancia de mantener un estilo de vida activo; en ese sentido, se deben fomentar cuidados preventivos en el personal docente; empezar con un control médico de salud integral e ir planificando con un profesional el tipo de actividad física acorde a la edad. Finalmente, se encuentra que hay incidencia de problemas de salud, por tanto, existe la necesidad de considerar la actividad física como elemento que coadyuva al mejoramiento de las condiciones de salud de los Docentes de la Unidad Educativa Atahualpa.

\section{Referencias bibliográficas.}

Barrera, R. (2017). Cuestionario Internacional de actividad física (IPAQ). Revista Enfermería del trabajo, 7(2), 49-54.

Barrón, C. (enero-abril, 2015). Concepciones epistemológicas y práctica docente. Una revisión. Redu. Revista de Docencia Universitaria,13(1), 35-56. doi:https://doi.org/10.4995/redu.2015.6436

Bonilla, E., \& Rodríguez, P. (2013). Más allá del dilema de los métodos. Bogotá: Ediciones Uniandes. Obtenido de https://es.scribd.com/document/375854141/BONILLA-RODRIGUEZ-Mas-AllaDel-Dilema-de-Los-Metodos-UPA

Cáceres, G. (2014). La importancia de publicar los resultados de Investigación. Revista Facultad de Ingeniería, 23(37), 7-8. Obtenido de https://www.redalyc.org/pdf/4139/413937008001.

Castro, E., \& Olmos, J. (2014). Características de las interacciones con la sociedad de los investigadores de humanidades y ciencias sociales a partir de estudios empíricos. Revista CTS, 27(9), 113-141. Obtenido de https://www.redalyc.org/pdf/924/92431880007.pdf

Cazau, P. (2011). Evolución de las relaciones entre la epistemología y la metodología de la investigación. Paradigmas. 3(1), 109-126. Obtenido de https://dialnet.unirioja.es/descarg a/articulo/3798214.pdf

Cuadarado, F. M. (2017). Efectividad de un programa de ejercicio físico sobre la calidad de vida relacionada con la salud de cuidadoras familiares de pacientes dependientes. Intervención desde fisioterapia de atención primaria(Doctoral dissertation, Universidad de Salamanca) 
Guevara-Manrique, A. C., Sánchez-Lozano, C. M., \& Parra, L. (2014). Estrés laboral y salud mental en docentes de primaria y secundaria. Revista colombiana de salud ocupacional, 4(4), 30-32.

Hidalgo Alava, D. J. (2017). Las pausas activas en el desempeño laboral de los servidores administrativos de la Universidad Técnica de Ambato (Bachelor's thesis, Universidad Técnica de Ambato. Facultad de Ciencias Humanas y de la Educación. Maestría en Cultura Física y Entrenamiento Deportivo).

Mocha-Bonilla Julio, A., Alava, D. J. H., Muñoz, J. M. V., \& Castro, N. J. V. (2018). OBESITY AND SEDENTARISM LEVELS ANALYSIS: A CASE STUDY UNIVERSIDAD TÉCNICA DE AMBATOINGAHURCO CAMPUS. International Journal of Sciences and Research, 74(5/1).

Montoya Marin, B. D. (2017). El estrés laboral y su relación con la calidad de vida de los empleados no docentes del Politécnico Colombiano Jaime Isaza Cadavid, sede Poblado, ciudad de Medellín.

OMS. Informe sobre la salud en el mundo 2014. Reducir los riesgos y promover una vida sana. Ginebra, 2014. Disponible en http://www.who.int/whr/2002/en/whr02_es.pdf.

Rodríguez, S., Núñez, J. C., Valle, A., Blas, R., \& Rosario, P. (2009). Auto-eficacia docente, motivación del profesor y estrategias de enseñanza. Escritos de Psicología (Internet), 3(1), 1-7.

Rodríguez Ramírez, J. A., Guevara Araiza, A., \& Viramontes Anaya, E. (2017). Síndrome de burnout en docentes. IE Revista de Investigación Educativa de la REDIECH, 8(14), 45-67.

Sánchez-González, J. L., Calvo-Arenillas, J. I., \& Sánchez-Rodríguez, J. L. (2018). Efectos del ejercicio físico moderado sobre la cognición en adultos mayores de 60 años. Revista de Neurología, 66(7), 230-236.

\section{【| Ciencia}




\section{PARA CITAR EL ARTÍCULO INDEXADO.}

Medina Paredes , S. V., Flores Robalino, R. G., Villalba Garzón, G. A., \& Barrera Cueva, J. del C. (2021). Identificación de problemas de salud como efecto del sedentarismo: un estudio con personas dedicadas a la docencia durante la pandemia covid19. ConcienciaDigital, $\quad 4(1.2)$, 457-469. https://doi.org/10.33262/concienciadigital.v4i1.2.1612

\section{Liencia}

El artículo que se publica es de exclusiva responsabilidad de los autores y no necesariamente reflejan el pensamiento de la Revista Conciencia Digital.

El artículo queda en propiedad de la revista y, por tanto, su publicación parcial y/o total en otro medio tiene que ser autorizado por el director de la Revista Conciencia Digital.
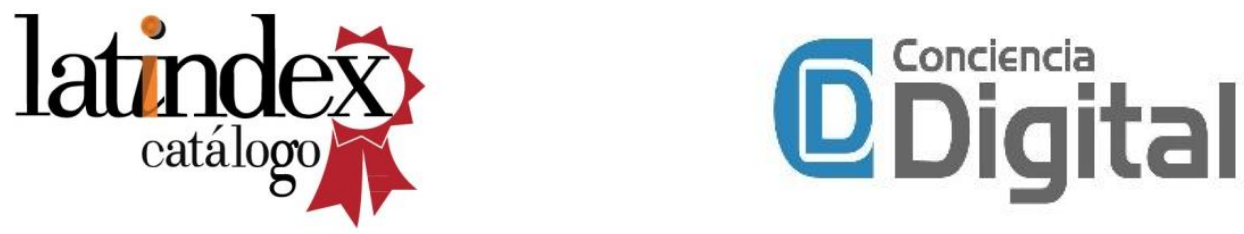